\title{
Several determinantal expressions of generalized Tribonacci polynomials and sequences
}

\author{
Can Kizilates ${ }^{1}$, Wei-Shih $\mathrm{Du}^{2}$, and Feng Qi ()$^{3}$ \\ ${ }^{1}$ Zonguldak Bulent Ecevit University \\ ${ }^{2}$ National Kaohsiung Normal University \\ ${ }^{3}$ Tianjin Polytechnic University
}

July 14, 2020

\begin{abstract}
In the paper, the authors present several explicit formulas for the (p, q, r)-Tribonacci polynomials and generalized Tribonacci sequences in terms of the Hessenberg determinants and, consequently, derive several explicit formulas for the Tribonacci numbers and polynomials, the Tribonacci-Lucas numbers, the Perrin numbers, the Padovan (Cordonnier) numbers, the Van der Laan numbers, the Narayana numbers, the third order Jacobsthal numbers, and the third order Jacobsthal-Lucas numbers in terms of special Hessenberg determinants.
\end{abstract}

\section{Hosted file}

Trib-Sym-S.pdf available at https://authorea.com/users/23442/articles/469363-severaldeterminantal-expressions-of-generalized-tribonacci-polynomials-and-sequences 